\title{
INTERFERÊNCIA DO TIPO DE MÁ OCLUSÃO NAS MEDIDAS DOS MOVIMENTOS MANDIBULARES: UM ESTUDO REALIZADO COM O APOIO DO EXÉRCITO BRASILEIRO
}

\author{
Interference of malocclusion types in mandibular movements \\ measures: a study supported by the Brazilian Army
}

\author{
Andrea Luiza Taumaturgo Metzger (1), Alcione Ramos Campiotto ${ }^{(2)}$, Paulo Cavalcante Muzy ${ }^{(3)}$
}

\section{RESUMO}

Objetivo: correlacionar os diferentes tipos de má oclusões dentais com as medidas de lateralidade e protrusão mandibular e amplitude de abertura bucal de indivíduos adultos normais. Métodos: neste estudo participaram 127 militares do sexo masculino que serviam no 21ํㅡㄹ Depósito de Suprimento do Exército Brasileiro em São Paulo, no ano de 2005, com idades entre 18 e 32 anos. Do total de militares, $47(39,16 \%)$ foram excluídos. Desse modo, a amostra final foi de 80 indivíduos os quais foram divididos em grupo controle (31-38,75\%) e grupo experimental $(49-61,25 \%)$. Na avaliação, foi realizada uma breve anamnese e inspeção oral, com posterior medição dos movimentos mandibulares de abertura bucal, protrusão e lateralidade para direita e para esquerda. Resultados: 69,38\% de indivíduos portadores da má oclusão Classe I de Angle; 16,32\% de Classe II-1; 6,12\% de Classe II-2; $8,16 \%$ de Classe III. A medida de abertura de boca, relacionada com os tipos de má oclusão, não foi estatisticamente significante, apesar de terem sido obtidas as maiores medidas nos indivíduos portadores de Classe III. Já nas medidas de protrusão e lateralidade mandibular existiu uma diferença estatisticamente significante nos grupos de uma maneira em geral. 40,42\% dos indivíduos apresentavam ausência de algum dente molar. Conclusão: não houve relação entre a medida de abertura de boca com a presença de má oclusões. Entretanto, houve relação entre as medidas de protrusão e de lateralidade mandibular para a direita e para a esquerda com má oclusões.

DESCRITORES: Má Oclusão de Angle Classe I; Má Oclusão de Angle Classe II; Má Oclusão de Angle Classe III; Medidas

\section{INTRODUÇÃO}

O sistema estomatognático é composto por ossos, dentes, articulação temporomandibular,

(1) Fonoaudióloga da Associação Fundo de Auxílio Mútuo dos Militares do Estado de São Paulo; Aperfeiçoada em Motricidade Orofacial pela Irmandade da Santa Casa de Misericórdia de São Paulo.

(2) Fonoaudióloga; Professora Assistente da Faculdade de Ciências Médicas da Santa Casa de São Paulo, FCMSCSP, São Paulo, SP; Coordenadora do Curso de Aperfeiçoamento em Motricidade Orofacial da Santa Casa de SP; Especialista em Voz e em Motricidade Orofacial; Mestre em Distúrbios da Comunicação Humana pela Pontifícia Universidade Católica de São Paulo.

(3) Oficial Médico Chefe da Seção de Saúde do 21을epósito de Suprimento do Exército Brasileiro no ano de 2005; Residente do serviço de Ortopedia e Traumatologia da Escola Paulista de Medicina; Especialista em Fisiologia do Exercício e Biomecânica do Exercício. músculos, sistema vascular, sistema nervoso e espaços vazios. Qualquer alteração, principalmente sobre os dentes, tenderá a levar a um desequilíbrio de todo o sistema ${ }^{1}$.

A relação entre os dentes é chamada de oclusão². Em 1899, Angle ${ }^{3}$ definiu que em uma oclusão normal, cada arco dentário descreve uma curva na qual os dentes da arcada superior devem estar posicionados em harmonia com seus adjacentes e também com os da arcada inferior. Portanto, a má oclusão pode ser definida como o arranjo ou a disposição dos dentes no arco dentário e a relação destes com as bases ósseas e as estruturas relacionadas de forma diferente daquela aceita como fórmula humana ${ }^{4}$, tendo sido classificada em classe I, II (divisão 1 e 2) e III por Angle ${ }^{3}$.

Para essa classificação, o primeiro molar permanente é o elemento dental mais importante, pois 
Angle o classificou como o "dente chave de oclusão" ${ }^{5}$. Esse é um dos motivos pelo qual este deva merecer toda atenção e cuidados, mas não só por sua importância funcional e necessidade de preservação, como também, por sua alta suscetibilidade à lesão de cárie e suas graves conseqüências, como a extração ${ }^{6}$.

As más oclusões podem levar a mudanças na posição das articulações temporomandibulares ${ }^{7}$. O mesmo autor constatou, por meio de um levantamento bibliográfico, que a posição anatômica da cabeça da mandíbula na fossa mandibular mais encontrada, em pesquisas realizadas, quando relacionadas às más oclusões são: Classe I (incluindo a oclusão considerada normal), centralizada; Classe II divisão 1, anteriorizada; Classe II divisão 2, posteriorizada; Classe III, anteriorizada.

Estas observações sugerem que pacientes portadores de más oclusões possam apresentar alterações na biodinâmica mandibular que poderá influenciar a realização dos movimentos de lateralidade e protrusão, assim como a própria amplitude de abertura bucal.

Para este estudo, serão comparadas as medidas mandibulares com os tipos de má oclusão, já que tais manifestações podem interferir na posição anatômica da cabeça da mandíbula na fossa mandibular ${ }^{7}$.

Portanto, o objetivo deste estudo é relacionar os diferentes tipos de má oclusões dentais (classificação de Angle $^{3}$ ) com medidas de lateralidade e protrusão mandibular e amplitude de abertura bucal em indivíduos adultos sem queixas miofuncionais orofaciais.

\section{MÉTODOS}

A amostra foi composta por 127 militares do sexo masculino de diferentes raças (brancos, negros e mulatos) que serviam no $21^{\circ}$ Depósito de Suprimento do Exército Brasileiro em São Paulo, no ano de 2005, com idades entre 18 e 32 anos. Do total, 47 foram excluídos devido à diferença da oclusão dentária quando comparado um lado com o outro; presença de alterações na mordida (aberta, topo a topo, cruzada) em nível dos dentes molares ou ausência destes.

Desse modo, a amostra foi de 80 indivíduos os quais foram divididos em grupo controle (31-38,75\%) e grupo experimental $(49-61,25 \%)$. Para fazer parte do primeiro grupo, era necessário que os participantes nunca tivessem realizado tratamento ortodôntico, mas que apresentassem uma oclusão chave-molar normal. Já o grupo experimental foi composto por soldados que também nunca haviam realizado tratamento ortodôntico, mas que apresentavam algum tipo de má oclusão pela classificação de Angle ${ }^{3}$.

Tais soldados foram selecionados aleatoriamente, apenas com a condição de não terem realizado tratamento ortodôntico prévio.

Todos os dados foram coletados por uma única examinadora, graduada em fonoaudiologia, apta a exercer a profissão pelo Conselho Regional de Fonoaudiologia/2a região.

Inicialmente, foi solicitado para que os participantes lessem o termo de consentimento de participação na pesquisa com posterior assinatura no espaço reservado.

Após essa etapa, foi realizada uma breve anamnese que continha dados de identificação dos sujeitos; preferência de lateralidade mastigatória (se a resposta fosse positiva, era perguntado o lado e o motivo) e preferência por uma determinada consistência de alimento (líquido, pastoso ou sólido).

Para a realização da inspeção oral foi pedido para que os participantes se posicionassem sentados em uma cadeira comum, com os pés apoiados no chão, coluna ereta e cabeça em posição ortostática.

Foram observados pela fonoaudióloga: tipo de oclusão (chave-molar pela classificação de Angle); estado de conservação dos dentes (BEC-bom estado de conservação ou MEC-mau estado de conservação); ausências dentárias; tipo de mordida (aberta; topo a topo; cruzada; presença de sobressaliência, mordida profunda, diastemas, apinhamento; desvio de linha média); sinais e/ou sintomas de disfunção temporomandibular na abertura e fechamento bucal.

Também foi realizada palpação dos músculos masseteres e temporais para verificação de dor e simetria de contração muscular. Esta foi realizada da seguinte maneira: com a avaliadora posicionada em frente ao participante com as mãos sobre os masseteres direito e esquerdo para percepção de simetria da contração muscular; após esta etapa, foi perguntado ao participante se durante a palpação houve algum sintoma de dor. Posteriormente, o mesmo procedimento foi realizado nos músculos temporais.

Para a medição dos movimentos mandibulares (abertura de boca, protrusão e lateralidade para direita e esquerda), foi utilizado um paquímetro digital da marca Digimess do fabricante Pró-Fono. A medida de abertura bucal foi realizada com o paquímetro posicionado entre os incisivos superiores e inferiores (distância interincisivos), pedindo para que o participante abrisse a boca ao máximo (abertura forçada). Já a medida de protrusão mandibular foi obtida através da solicitação da avaliadora para que o participante protruísse a mandíbula 
para frente ao máximo. Por fim, foi obtida a medida de lateralidade mandibular para a direita e para a esquerda. Essa medição foi feita com o paquímetro posicionado através das linhas médias dos incisivos superiores e inferiores.

Todos os participantes deste estudo (grupo controle e experimental) foram avaliados com o auxílio de um protocolo elaborado pela avaliadora responsável especificamente para este estudo.

A pesquisa recebeu parecer favorável do CEP Comitê de Ética em Pesquisa do Hospital Central da Irmandade da Santa Casa de Misericórdia de São Paulo, sob o número 031/05.

Para a análise estatística deste estudo foram usados os testes Kruskal-Wallis e Mann-Whitney e um nível de significância de 0,10.

\section{RESULTADOS}

Do total de 127 indivíduos avaliados neste estudo, $47(39,16 \%)$ foram excluídos da amostra. Destes 47, 19 (40,42\%) indivíduos tinham extraído algum molar superior, inferior ou ambos. $O$ restante (28 - 59,57\%) foi excluído por alteração da mordida nos dentes molares ou diferença da oclusão chavemolar quando comparado um lado com o outro.

Portanto, foram analisados, neste estudo, 80 militares que foram divididos em grupo controle (31-38,75\%) e grupo experimental $(49-61,25 \%)$,

Dentro do grupo experimental, os indivíduos foram separados em: Classe I (34-69,38\%), Classe II-1 (8-16,32\%), Classe II-2 (3-6,12\%) e Classe III (4-8,16\%). Portanto, o tipo de má oclusão mais encontrada neste estudo foi a Classe I $(69,38 \%)$. Tais dados são observados na Figura 1.

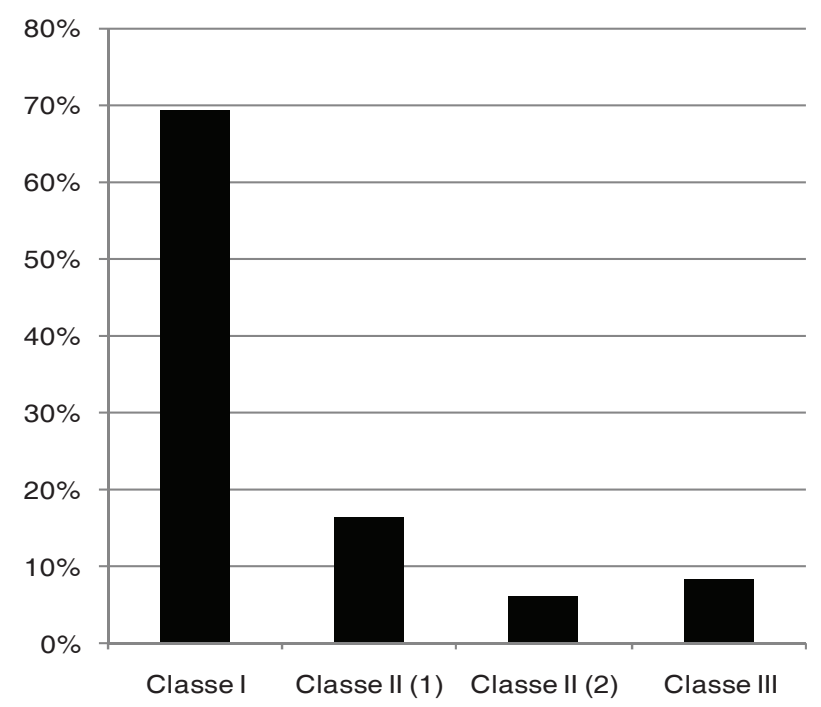

Figura 1 - Distribuição dos sujeitos quanto ao tipo de má oclusão
A partir da análise estatística, foi definido para este trabalho um nível de significância de 0,10 $(10 \%)$.

Ressalta-se que foram utilizados testes e técnicas estatísticas não paramétricas, porque as condições para a utilização de técnicas e testes paramétricos, como a normalidade e homocedasticidade não foram encontradas (principalmente a normalidade) neste conjunto de dados obtidos.

Ao analisar a medida de abertura de boca (AB) com o auxílio do teste de Kruskal-Wallis para comparar os resultados entre os grupos Classe I, Classe II-1, Classe II-2, Classe III e Normal (Tabela 1), observa-se que a média de abertura de boca nos diferentes tipos de má oclusão foi: Classe I (50,52 mm); Classe II-1 (51,88 mm); Classe II-2 $(44,67 \mathrm{~mm})$; Classe III (55 mm). Portanto, a maior média encontrada foi nos indivíduos portadores da má oclusão Classe III. Entretanto, tais resultados não são considerados estatisticamente significantes. Os indivíduos do grupo controle obtiveram uma média de 50,61 mm.

Ao analisar a medida de protrusão mandibular (Tabela 2), pode-se observar que a média de protrusão mandibular nos diferentes tipos de má oclusão foi: Classe I (5,71 mm); Classe II-1 (5,25 mm); Classe II-2 (5,33 mm); Classe III (5,25 mm). Os indivíduos do grupo controle obtiveram uma média de $7 \mathrm{~mm}$.

Analisando tal tabela, conclui-se que existe diferença estatisticamente significante entre os grupos de uma maneira em geral. Porém, é preciso especificar onde a diferença ocorre de fato. Sendo assim, utilizou-se o teste de Mann-Whitney para comparar todos os grupos dois na dois e foi apresentado na Tabela 3 somente os $p$-valores destas comparações.

Nas medidas de lateralidade também existiu uma diferença estatisticamente significante entre os grupos de uma maneira em geral tanto para a direita (Tabela 4) quanto para a esquerda (Tabela 5). Sendo assim, utilizou-se o teste de Mann-Whitney (Tabela 6 e 7) para comparar todos os grupos dois a dois e descobrir onde a diferença ocorre de fato. O segundo quadro mostra somente os $p$-valores destas comparações.

As médias de lateralidade mandibular para a direita e para a esquerda, respectivamente, foram: Classe I (10 mm-9,21 mm); Classe II-1 (7,75 mm$11,63 \mathrm{~mm})$; Classe II-2 (10 mm-11,67 mm); Classe III $(8,75 \mathrm{~mm}-8,25 \mathrm{~mm})$. Os indivíduos com oclusão normal obtiveram uma média de $9,48 \mathrm{~mm}$ para a direita e 10,19 $\mathrm{mm}$ para esquerda. 
Tabela 1 - Distribuição dos resultados referentes às medidas de abertura de boca, de acordo com a classificação da má oclusão

\begin{tabular}{lccccc}
\hline AB & Classe I & Classe II-1 & Classe II-2 & Classe III & Normal \\
\hline Média & 50,82 & 51,88 & 44,67 & 55,00 & 50,61 \\
Mediana & 50 & 53,5 & 45 & 55,5 & 52 \\
Desvio Padrão & 6,65 & 5,62 & 1,53 & 4,55 & 6,30 \\
Quartil 1 & 46 & 50,5 & 44 & 53,5 & 46,5 \\
Quartil 3 & 56 & 55,5 & 45,5 & 57 & 55 \\
Tamanho & 34 & 8 & 3 & 4 & 31 \\
Intervalo de Confiança & 2,23 & 3,89 & 1,73 & 4,46 & 2,22 \\
\hline p-valor & & 0,205 & & \\
\hline
\end{tabular}

Teste estatístico utilizado: Kruskall-Wallis. Nível de Significância: $p<0,10$

Tabela 2 - Distribuição dos resultados referentes às medidas de protrusão mandibular, de acordo com a classificação da má oclusão

\begin{tabular}{lccccc}
\hline Protrusão & Classe I & Classe II-1 & Classe II-2 & Classe III & Normal \\
\hline Média & 5,71 & 5,25 & 5,33 & 5,25 & 7,00 \\
Mediana & 5 & 3,5 & 4 & 5,5 & 6 \\
Desvio Padrão & 2,14 & 4,53 & 2,31 & 2,50 & 2,22 \\
Quartil 1 & 4,25 & 3 & 4 & 4,25 & 5 \\
Quartil 3 & 7 & 5,25 & 6 & 6,5 & 9 \\
Tamanho & 34 & 8 & 3 & 4 & 31 \\
Intervalo de Confiança & 0,72 & 3,14 & 2,61 & 2,45 & 0,78 \\
\hline p-valor & & & $\mathbf{0 , 0 3 3}^{*}$ & & \\
\hline
\end{tabular}

Teste estatístico utilizado: Kruskall-Wallis. Nível de Significância: $p<0,10$

Tabela 3 - Distribuição das medidas de protrusão mandibular, de acordo com a classificação da má oclusão

\begin{tabular}{lcccc}
\hline Protrusão & Classe I & Classe II-1 & Classe II-2 & Classe III \\
\hline Classe II-1 & $0,110 \#$ & & & \\
Classe II-2 & 0,629 & 0,406 & & \\
Classe III & 0,903 & 0,547 & 0,857 & \\
Normal & $0,018^{*}$ & $0,015^{\star}$ & $0,146 \#$ & 0,234 \\
\hline
\end{tabular}

Teste estatístico utilizado: Mann-Whitney. Nível de Significância: $p<0,10$

Tabela 4 - Distribuição dos resultados referentes às medidas de lateralidade mandibular para a direita, de acordo com a classificação da má oclusão

\begin{tabular}{lccccc}
\hline Direita & Classe I & Classe II-1 & Classe II-2 & Classe III & Normal \\
\hline Média & 10,00 & 7,75 & 10,00 & 8,75 & 9,48 \\
Mediana & 10 & 7,5 & 10 & 8,5 & 10 \\
Desvio Padrão & 2,12 & 2,55 & 0,00 & 1,71 & 1,86 \\
Quartil 1 & 9 & 6,5 & 10 & 7,75 & 8,5 \\
Quartil 3 & 11 & 8,25 & 10 & 9,5 & 10,5 \\
Tamanho & 34 & 8 & 3 & 4 & 31 \\
Intervalo de Confiança & 0,71 & 1,77 & $-\mathbf{x}-$ & 1,67 & 0,65 \\
\hline p-valor & & & $\mathbf{0 , 0 7 5}$ & & \\
\hline
\end{tabular}

Teste estatístico utilizado: Kruskall-Wallis. Nível de Significância: $p<0,10$ 
Tabela 5 - Distribuição dos resultados referentes às medidas de lateralidade mandibular para a esquerda, de acordo com a classificação da má oclusão

\begin{tabular}{lccccc}
\hline Esquerda & Classe I & Classe II-1 & Classe II-2 & Classe III & Normal \\
\hline Média & 9,21 & 11,63 & 11,67 & 8,25 & 10,19 \\
\hline Mediana & 9,5 & 12 & 13 & 8,5 & 10 \\
\hline Desvio Padrão & 2,68 & 2,20 & 2,31 & 1,71 & 1,49 \\
\hline Quartil 1 & 8,25 & 10,5 & 11 & 7,5 & 9 \\
\hline Quartil 3 & 10 & 13,25 & 13 & 9,25 & 11 \\
\hline Tamanho & 34 & 8 & 3 & 4 & 31 \\
\hline Intervalo de Confiança & 0,90 & 1,52 & 2,61 & 1,67 & 0,53 \\
\hline p-valor & & & $\mathbf{0 , 0 2 7 ^ { * }}$ & & \\
\hline
\end{tabular}

Teste estatístico utilizado: Kruskall-Wallis. Nível de Significância: $p<0,10$

Tabela 6 - Distribuição das medidas de lateralidade mandibular para direita, de acordo com a classificação da má oclusão

\begin{tabular}{lcccc}
\hline Direita & Classe I & Classe II-1 & Classe II-2 & Classe III \\
\hline Classe II-1 & $0,010^{*}$ & & & \\
Classe II-2 & 0,798 & $0,062^{*}$ & & \\
Classe III & 0,201 & 0,342 & 0,271 & \\
Normal & 0,462 & $0,026^{*}$ & 0,638 & 0,370 \\
\hline
\end{tabular}

Teste estatístico utilizado: Mann-Whitney. Nível de Significância: $p<0,10$

Tabela 7 - Distribuição das medidas de lateralidade mandibular para a esquerda, de acordo com a classificação da má oclusão

\begin{tabular}{lcccc}
\hline Esquerda & Classe I & Classe II-1 & Classe II-2 & Classe III \\
\hline Classe II-1 & $0,024^{*}$ & & & \\
Classe II-2 & $0,141 \#$ & 0,917 & & \\
Classe III & 0,276 & $0,040^{*}$ & $0,105^{*}$ & \\
Normal & $0,126 \#$ & $0,071^{*}$ & 0,225 & $0,046^{*}$ \\
\hline
\end{tabular}

Teste estatístico utilizado: Mann-Whitney. Nível de Significância: $p<0,10$

\section{DISCUSSÃO}

Nesse trabalho foi observada uma prevalência de $40,42 \%$ de indivíduos com ausência de algum dos dentes molares. Em outro estudo ${ }^{8}, 36,7 \%$ dos indivíduos do sexo masculino havia extraído algum dente permanente, sendo que $91,8 \%$ eram dentes posteriores.

Nas cidades de Maceió e Recife ${ }^{9}$, também houve uma maior porcentagem de perda dos dentes posteriores, $65,8 \%$ e $83,6 \%$ respectivamente. Estes resultados mostrados são maiores do que os obtidos neste estudo. A cárie foi apontada como o principal motivo das extrações dentárias.

Estudos internacionais também mostram a cárie como a principal causa de extração ${ }^{10,11}$.
Já em outro trabalho ${ }^{12}$, a porcentagem de dentes ausentes foi de $3,48 \%$. Este dado não é semelhante à presente pesquisa, talvez pela diferença na faixa etária entre ambos, já que no primeiro foi realizado dos seis aos 18 anos e este dos 18 aos 32 anos.

A manutenção dos primeiros molares permanentes na arcada dental é de fundamental importância para o estabelecimento de uma oclusão estável, pois eles constituem, de forma específica, parte essencial do sistema estomatognático ${ }^{6}$.

Dentro do grupo das más oclusões, o tipo mais encontrado, neste estudo, foi a Classe I (69,38\%).

Em um outro realizado em $2002{ }^{13}$, foram encontrados: $48 \%$ de indivíduos portadores de má oclusão Classe I, $42 \%$ de Classe II e 3\% de Classe III. Outro realizado também no ano de $2002{ }^{4}$ foi encontrado: $41 \%$ de portadores de Classe I, $26 \%$ de Classe II e 
$8 \%$ de Classe III. Em ambas as pesquisas, os resultados foram compatíveis com os apresentados, pois o tipo de má oclusão Classe I predominou na amostra.

Estudos internacionais mostram diferentes resultados com relação à prevalência dos tipos de má oclusão, como por exemplo, em alguns realizados em países da África mostram resultados compatíveis, ou seja, maior prevalência da má oclusão Classe I. Essa compatibilidade pode ser explicada pela semelhança do tipo racial quando comparado ao do brasileiro ${ }^{14,15}$. Já em um norte-americano, foi mostrada uma maior prevalência do tipo de má oclusão Classe II ${ }^{16}$.

Com relação às medidas mandibulares, a abertura de boca nos indivíduos portadores de má oclusão Classe III foi a maior medida encontrada. Entretanto, não foi estatisticamente significante.

A média encontrada da medida de abertura de boca nos indivíduos com oclusão normal foi de $50,61 \mathrm{~mm}$. Em outra pesquisa ${ }^{17}$, foi encontrada uma média de abertura de boca em homens de $45,13 \mathrm{~mm}$. Este valor é menor do que o encontrado neste estudo. Esta diferença talvez tenha ocorrido pelo fato da nossa amostra ter sido composta, principalmente, por indivíduos negros e mulatos, ou seja, por indivíduos que apresentam uma maior abertura de boca quando comparados a outras raças.

Um trabalho realizado na França mostrou resultado muito semelhante à presente pesquisa. Mostrou uma média de abertura de boca em indivíduos adultos de $50,77 \mathrm{~mm}^{18}$. Já em outro realizado também na Europa ${ }^{19}$, a média de abertura de boca foi de $50,6 \mathrm{~mm}$, ou seja, o mesmo resultado.

$\mathrm{Na}$ medida de protrusão mandibular, foi encontrado um resultado estatisticamente significante nos grupos de uma maneira em geral. A média desta medida, nos indivíduos portadores de oclusão normal, foi de $7 \mathrm{~mm}$. Tal resultado é compatível com o Índice de Disfunção Clínica de Helkimo e o Índice Craniomandibular ${ }^{20}$, os quais também mostram como normalidade protrusão mandibular de $7 \mathrm{~mm}$. Além disso, um trabalho internacional mostrou um valor de $8,2 \mathrm{~mm}$, ou seja, maior do que o obtido no presente estudo ${ }^{19}$.
Nas medidas de lateralidade para a direita $e$ para a esquerda, também foram encontrados resultados estatisticamente significantes nos grupos de uma maneira em geral.

Nos indivíduos portadores de oclusão normal, as médias encontradas das medidas de lateralidade para a direita e para a esquerda foram de $9,48 \mathrm{~mm}$ e $10,19 \mathrm{~mm}$, respectivamente. Estudo internacional mostrou semelhança nos resultados: $10,2 \mathrm{~mm}$ para a direita e $10,6 \mathrm{~mm}$ para a esquerda ${ }^{19}$. Entretanto, não foram compatíveis com Índice de Disfunção Clínica de Helkimo e o Índice Craniomandibular ${ }^{20}$, já que estes demonstram como normalidade uma medida de $7 \mathrm{~mm}$ tanto para a direita como para a esquerda. Portanto, sugere-se que os dados de literatura devam ser revistos para a nossa população.

É importante salientar que não foram encontrados trabalhos nacionais nem internacionais relacionando os tipos de má oclusão com as medidas mandibulares. Portanto, não foi possível comparar os dados obtidos neste estudo com de outros autores. Sugere-se a realização de mais trabalhos que abranjam este tema.

\section{CONCLUSÃO}

A partir da análise dos dados pode-se concluir que não houve relação entre a medida de abertura de boca e a presença de má oclusões. Entretanto, houve relação entre as medidas de protrusão e lateralidade mandibular para direita e esquerda com as más oclusões.

\section{AGRADECIMENTOS}

Agradecemos ao $21^{\circ}$ Depósito de Suprimentos do Exército Brasileiro e a todas pessoas que contribuíram para a realização deste trabalho: Carlos Euclides Olschowsky (Coronel Intendente), José Luiz Monteiro Giambartholomei (Tenente Coronel Intendente), Marcus Fernando Camillo Galia (Tenente Coronel Intendente), equipe da Seção de Saúde e a todos participantes da amostra. 


\section{ABSTRACT}

Purpose: to establish the relationship between different types of dental malocclusions and lateralization and protrusion measures and mouth opening in normal adult subjects. Methods: it was made with 127 military men who served at the $21^{\text {st }}$ Supply Deposit of Brazilian Army, in Sao Paulo, in 2005, with ages between 18 and 32 years. 47 subjects were excluded. Because of that, the final sample counted with 80 individuals whom has been divided in two groups: control group $(31-38.75 \%)$ and experimental group $(49-61.25 \%)$. On the evaluation, both a brief anamnesis and oral inspection with measurement of the mandibular movements (mouth opening, protrusion and right and left lateralization) were done. Results: $69.38 \%$ malocclusion Class I: $16.32 \%$ malocclusion Class II-1; $6.12 \%$ malocclusion Class II2; $8.16 \%$ malocclusion Class III. There was no statistical significance of the mouth opening measures, related with the types of malocclusion, although greater measures had been noted on subjects with Class III malocclusion. When comparing to protrusion and lateralization measures, we noticed a statistically relevance in all groups. There was an absence of grinders in $40.42 \%$ of the subjects. Conclusion: there was no relationship between different types of dental malocclusions and mouth opening measures, but there was relationship between different types of dental malocclusions and lateralization and protrusion measures.

KEYWORDS: Malocclusion Angle Class I; Malocclusion Angle Class II; Malocclusion Angle Class III; Measures

\section{REFERÊNCIAS}

1. Marchesan IQ. Avaliando e tratando o sistema estomatognático. In: Lopes Filho. Tratado de fonoaudiologia. São Paulo: Roca; 1997.

2. Sicher H, Dubrul EL. Anatomia oral por E. Loyd Dubrul. 8. ed. São Paulo: Artes Médicas; 1981.

3. Angle EH. Classification of malocclusion. Dental Cosmos. 1899; 41(2):248-65.

4. França BHS, Orellana B, Fronza F, Kowalski RV. Prevalência de maloclusão em pré-escolares de uma região da cidade de Curitiba. Rev Odonto Ciênc. 2002; 17(37):273-5.

5. Normando ADC, Silva MC, Lê Bihan R, Simone JL. Alterações oclusais espontâneas decorrentes da perda dos primeiros molares permanentes inferiores. Rev Dental Press Ortod Ortop Facial. 2003; 8(3):15-23.

6. Aguiar SMHCA, Santos Pinto RS. Lesões cariosas, restaurações e extrações por processo carioso de primeiros molares permanentes: estudo clínico e radiográfico. Rev Odontol UNESP. 1996; 25(2):345-55.

7. Cunha MANR. Posição anatômica da cabeça da mandíbula na fossa mandibular nas más oclusões de classes I, II e III de Angle. [dissertação]. São Paulo (SP): Universidade Federal de São Paulo; 2002.

8. Silveira RCJ, Souza EHA, Caldas Júnior AF. Razões para extração de dentes permanentes. Odont Clin Cie. 2003; 1(3):207-10.
9. Silveira RCJ, Caldas Júnior AF, Souza EHA, Gusmão ES. Razões das perdas dentárias nas cidades de Maceió e Recife, Brasil. Arq Odontol. 2004; 40(3):229-36.

10. Franklin D. Extraction of first permanent molars. Br Dent J. 2007; 203(7):408-9.

11. Bagain ZH, Khraisat A, Sawair F, Ghanam $S$, Shaini FJ, Rajab LD. Dental extraction for patients presenting at oral surgery student clinic. Compend Contin Educ Dent. 2007; 28(3):146-50.

12. Coser MC, Coser RM, Chiavini P, Boeck EM, Vedovello $S$, Lucato $A S$. Freqüência de cárie e perda dos primeiros molares permanentes: estudo em pacientes assistidos na clínica integrada infantil. RGO. 2005; 53(1):63-6.

13. Reis SAB, Capellozza Filho L, Mandetta S. Prevalência de oclusão normal e má oclusão em brasileiros, adultos, leucodermas, caracterizados pela normalidade do perfil facial. Rev Dental Press Ortodon Ortop Facial. 2002; 7(5):17-25.

14. Onyeaso CO, Aderinokun GA, Arowojolu MO. The pattern of malocclusion among orthodontic patientes seen in Dental Centre, University College Hospital, Ibadan, Nigeria. Afr J Med Med Sci. 2002; 31(3):207-11.

15. Onyeaso CO. Prevalence of malocclusion among adolescents in Ibadan, Nigéria. Am J Orthod Dentofacial Orthop. 2004; 126(5):604-7.

16. Profitt WR, Fields HW, Moray LJ. Prevalence of malocclusion and orthodontic treatment need in the United States: estimates from NHANES III survey. Int J Adult Orthod Orthog Surg. 1998; 13(2):97-106. 
17. Fogaça CL. Abertura máxima de boca: estudo clínico - método direto. J Bras Odontol Clin. 1998; 2(12):27-30.

18. Placko G, Bellot-Samson V, Brunet S, Guyot L, Richard O, Cheynet F, et al. [Normal mouth opening in the adult French population]. Rev Stomatol Chir Maxillofac. 2005; 106(5):267-71.

19. Hirsch C, John MT, Lautenschläger C, List T.
Mandibular jaw movemente capacity in 10-17-yr-old children and adolescents: normative values and the influence of gender, age and temporomandibular disorders. Eur J Oral Sci. 2006; 114(6):465-70. 20. Pirolla ILM. Comparação entre medidas lineares e angulares da abertura bucal máxima. [dissertação]. São Paulo (SP): Universidade Federal de São Paulo; 2003.

DOI: 10.1590 / S1516-18462008005000007

RECEBIDO EM: 22/08/2007

ACEITO EM: 26/05/2008

Endereço para correspondência:

Andrea Luiza Taumaturgo Metzger

Rua Haddock Lobo, 1170 ap. 41

São Paulo - SP

CEP: 01414-002

E-mail: deaepm@yahoo.com.br 